\title{
Identification of childhood acute respiratory infection hospital admissions using International Classification of Diseases-Australian Modification (ICD-10-AM) codes
}

\author{
Marisa van Arragon MHealthMgt ${ }^{1}$, Andrew Wooding GradDipBus (Hlthlnfo) ${ }^{2}$ and Cameron C. Grant PhD ${ }^{1,3}$ (1) \\ ${ }^{1}$ Department of Paediatrics, University of Auckland, Auckland, New Zealand, ${ }^{2}$ Clinical Coding Department, Auckland District Health Board, Auckland, \\ New Zealand and ${ }^{3}$ General Paediatrics, Starship Children's Hospital, Auckland, New Zealand
}

To the Editor - The International Classification of Diseases (ICD) can be used to identify acute respiratory infection (ARI) hospital admissions. The coding system, used internationally to translate disease text descriptions from words into alphanumeric codes, contains 22 chapters $^{1}$ organized by epidemic and general diseases arranged by organ system, developmental diseases, and injuries. For example, Chapter X contains codes for the respiratory system in which code block J00-J06 describes acute upper respiratory infections and J09-J18 refers to influenza and pneumonia.

Alongside various benefits of this coding system are some limitations. Not all diseases of an organ system are coded in the chapter assigned to that system. This is the case for ARI codes, which are described mostly in Chapter X, with some in Chapter I, "Certain Infectious and Parasitic Diseases" and others in Chapter VIII "Diseases of the Ear and Mastoid Process." Therefore, we have described a methodology to identify all ICD-10 codes representing childhood ARIs.

\section{Methods}

We adapted an approach developed initially by the United States Centers for Disease Control and Prevention, subsequently modified by Baker et $\mathrm{al}^{2}{ }^{2}$ for the description of infectious diseases hospitalizations in New Zealand. Bakers' diagnostic group codes were selected for eye infections, ear infections, upper respiratory tract infections, tuberculosis, acute lower respiratory tract infections, chronic lower respiratory tract infections, other bacterial infections, and other viral infections. A second list of ICD-10 codes, developed by Saraf, ${ }^{3}$ who identified ARI hospital events in New Zealand occurring during the first year of life, was also utilized. From these studies, 611 ICD-10 2- and 3 -character codes were identified with respective ICD-10-AM descriptions. ${ }^{4}$

We considered 2 questions for each code/disease description. First, "Is this code/disease/health problem a respiratory infection?" The answer options included the following: always, predominantly, some cases, not a respiratory infection, or respiratory

Author for correspondence: Cameron Grant, E-mail: cc.grant@auckland.ac.nz PREVIOUS PRESENTATION. This study was presented in part at the Children's Inpatient Research Collaboration AUS/NZ Virtual Symposium on October 30, 2020.

Cite this article: van Arragon M, Wooding A, and Grant CC. (2022). Identification of childhood acute respiratory infection hospital admissions using International Classification of Diseases-Australian Modification (ICD-10-AM) codes. Infection Control \& Hospital Epidemiology, 43: 1281-1282, https://doi.org/10.1017/ice.2021.267 transmission but not a respiratory disease. Second, we asked, "Is this coded respiratory infection acute or chronic?" Here, the answer options were acute, chronic, both, or unknown.

Codes were reviewed independently by 2 clinician researchers (M.v.A. and C.C.G.). Lists were compared and consensus was reached by review of online clinical decision-support resources. ${ }^{5}$ Nonrespiratory conditions and chronic duration conditions were removed. Next, the resulting code list was compared with original lists from Baker et $\mathrm{al}^{2}$ and Saraf. ${ }^{3}$ We then re-evaluated discrepancies in ARI and acuity categorizations and justified the inclusion or exclusion of codes.

A subset of codes, blocks B95-B97, could not be classified. These were infectious agents, rather than disease codes, utilized alongside primary coding when identification of the causative agent is desired. ${ }^{1}$ With supervision from an expert clinical coder (A.W.), the clinical researchers learned basic hospital event coding to better understand the process. Two pilot tests, containing 85 and 93 hospital events respectively, were coded individually, compared, and discussed. Here, blocks B95-B97, representing infectious agents, alongside codes from "Chapter XVIII, Symptoms, Signs and Abnormal Clinical and Laboratory Findings" were used to code hospital events in which symptom-related discharge diagnoses had been recorded (eg, viralinduced wheeze). When a symptom code, R06.2-Wheezing, is combined with an infectious agent code such as B97.0Adenovirus, the combined codes identify an ARI hospital admission. Of 42 symptom codes from Chapter XVIII block R00-R09, 3 symptom codes (ie, R05-Cough, R06.1-Stridor and R06.2Wheezing) identify as such.

\section{Results}

Table 1 presents the list of 332 ICD-10-AM ${ }^{4}$ codes that represent a childhood ARI hospital admission. Codes from 5 ICD chapters were included. Codes in "Chapter X, Diseases of the Respiratory System" accounted for 97 (29\%) of 332 codes.

\section{Discussion}

This methodology was developed to identify ICD-10-AM codes that represent ARI hospital admissions in early childhood. It can be followed and adapted by others aiming to utilize the ICD coding system for disease-category enquiries. When developing a similar process, 3 aspects should be considered. First, published ICD-10 code lists should be scrutinized prior to applying them to a disease category of interest because motives for selecting codes for a disease category of interest are likely to be specific to the research question. Second, researchers should learn basic coding skills; they 
Table 1. International Classification of Diseases-Australian Modification (ICD-10-AM), Eighth Edition, Codes Identified as Representing Early Childhood Acute Respiratory Infection ARI Hospital Admissions

\begin{tabular}{|c|c|}
\hline ICD-10 Chapter & ICD-10-AM Alphanumeric Code \\
\hline $\begin{array}{l}\text { Chapter I, } \\
\text { Certain Infectious and Parasitic Diseases }\end{array}$ & 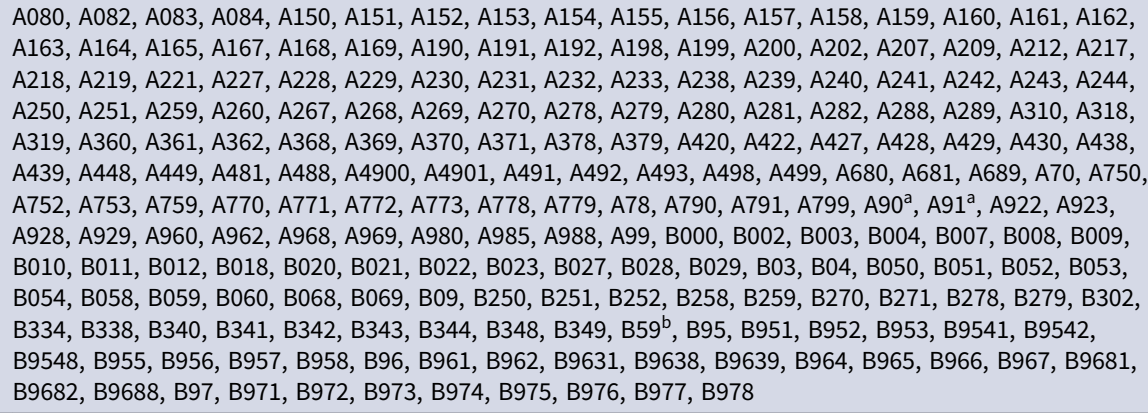 \\
\hline Chapter VII, Diseases of the Eye and Adnexa & $\mathrm{H} 042, \mathrm{H} 043$ \\
\hline $\begin{array}{l}\text { Chapter VIII, Diseases of the Ear and Mastoid } \\
\text { Process }\end{array}$ & $\begin{array}{l}\text { H650, H651, H659, H660, H664, H669, H670, H671, H678, H720, H721, H722, H728, H729, H730, H738, } \\
\text { H739 }\end{array}$ \\
\hline Chapter X, Diseases of the Respiratory System & 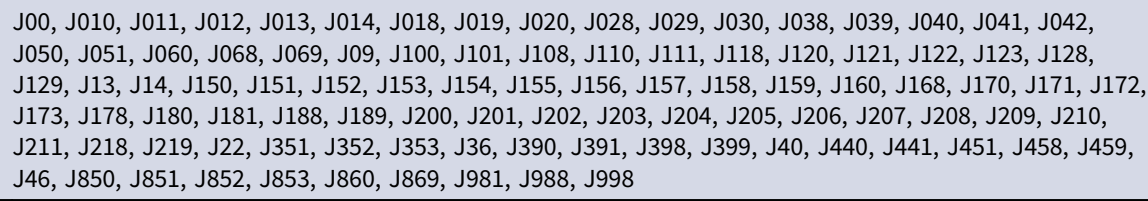 \\
\hline $\begin{array}{l}\text { Chapter XVIII, Symptoms, Signs, and Abnormal } \\
\text { Clinical and Laboratory Findings }\end{array}$ & R05, R061, R062 \\
\hline
\end{tabular}

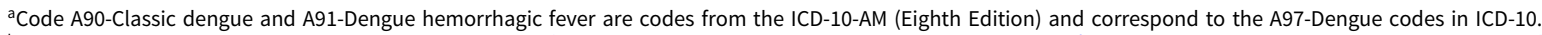

${ }^{\mathrm{b}}$ Code B59-Pneumocystosis in ICD-10-AM (Eighth Edition) ${ }^{4}$ corresponds to code B59 in the ICD-10 (2016 version), ${ }^{6}$ and to code B48.5 in the ICD-10 (2019 version). ${ }^{7}$

should attempt coding in addition to consulting coding experts. By doing so, researchers can more accurately utilize coding systems and rules relevant to the area being studied (eg, combined symptom/infectious agent codes). Third, researchers should consider country-specific ICD coding practices and base classification adaptations; for example, ICD-10-AM differs slightly from ICD-10.

This study has several limitations. First, despite the robust process followed, it was difficult to determine when all relevant ARI codes had been selected. We suggest additionally considering relevant blocks of codes from the ICD base classification itself alongside the inclusion of previously published lists of codes. Second, the frequent update of ICD classifications limits the generalizability of the resulting ARI codes list.

In conclusion, we have described a process with which to identify ICD codes for ARI hospital admissions during childhood. This approach can be applied to studies seeking to use ICD codes to describe hospital admissions for other specific disease categories.

Financial support. No financial support was provided relevant to this article.

Conflicts of interest. All authors report no conflicts of interest relevant to this article.

\section{References}

1. World Health Organization. International Statistical Classification of Diseases and Related Health Problems, Tenth Revision, Fifth Edition. World Health Organization website. https://icd.who.int/browse10/Content/ statichtml/ICD10Volume2_en_2019.pdf. Published 2016. Accessed June 5, 2021.

2. Baker MG, Barnard LT, Kvalsvig A, et al. Increasing incidence of serious infectious diseases and inequalities in New Zealand: a national epidemiological study. Lancet 2012;379:1112-1119.

3. Saraf R. Acute respiratory tract infections and vitamin D: neonatal vitamin D levels and acute respiratory tract infections in the first year of life. University of Auckland website. http://hdl.handle.net/2292/34853. Published 2017. Accessed June 5, 2021.

4. National Casemix and Classification Centre. The International Statistical Classification of Diseases and Related Health Problems, 10th Revision, Australian Modification (ICD-10-AM). Eight Edition. Darlinghurst, NSW: National Casemix and Classification Centre, UoW; 2013.

5. The clinical answers you need- anytime, anywhere. UpToDate website https://www.uptodate.com/home. Published 2020. Accessed June 5, 2021.

6. World Health Organization. ICD-10 browser version: 2016. World Health Organization World Health Organization website. https://icd.who.int/ browse10/2016/en. Published 2016. Accessed January 2021.

7. World Health Organization. ICD-10 browser version 2019. World Health Organization website. https://icd.who.int/browse10/2019/en. Published 2019. Accessed January 2021. 